\title{
Customization of Java Library Classes using Type Constraints and Profile Information
}

\author{
Bjorn De Sutter ${ }^{1}$, Frank Tip ${ }^{2}$, and Julian Dolby ${ }^{2}$ \\ 1 Ghent University, Electronics and Information Systems Department \\ Sint-Pietersnieuwstraat 419000 Gent, Belgium \\ brdsutte@elis.ugent.be \\ ${ }^{2}$ IBM T.J. Watson Research Center, P.O. Box 704, Yorktown Heights, NY 10598 \\ \{ ftip, dolby \}ous.ibm.com
}

\begin{abstract}
The use of class libraries increases programmer productivity by allowing programmers to focus on the functionality unique to their application. However, library classes are generally designed with some typical usage pattern in mind, and performance may be suboptimal if the actual usage differs. We present an approach for rewriting applications to use customized versions of library classes that are generated using a combination of static analysis and profile information. Type constraints are used to determine where customized classes may be used, and profile information is used to determine where customization is likely to be profitable. We applied this approach to a number of Java applications by customizing various standard container classes and the omnipresent StringBuffer class, and measured speedups up to $78 \%$ and memory footprint reductions up to $46 \%$. The increase in application size due to the added custom classes is limited to $12 \%$ for all but the smallest programs.
\end{abstract}

\section{Introduction}

The availability of a large library of standardized classes is an important reason for Java's popularity as a programming language. The use of class libraries improves programmer productivity by allowing programmers to focus on the aspects that are unique to their application without being burdened with the unexciting task of building (and debugging!) standard infrastructure. However, library classes are often designed and implemented with some typical usage pattern in mind. If the actual use of a library class by an application differs substantially from this typical usage pattern, performance may be suboptimal.

Consider, for example, the implementation of the container classes such as Vector and Hashtable in package java.util. In designing the implementation of these containers, a large number of accesses to objects stored therein was (implicitly) assumed. Therefore, the allocation of auxiliary data structures encapsulated by the container (e.g., a Vector's underlying array, or a Hashtable's embedded array of hash-buckets) is performed eagerly upon construction of the container itself. This approach has the advantage that the container's access methods can assume that these auxiliary data structures have been allocated. 
However, as we shall see in Section 6, it is not uncommon for programs to create large numbers of containers that remain empty or that contain only small numbers of objects. In such cases, lazy allocation is preferable, despite the fact that the access methods become slower because they have to check if the auxiliary data structures have been allocated and create them if this is not the case.

Library classes may also induce unnecessary overhead if an application does not use all of the provided functionality. For example, most iterators provided by containers such as Hashtable are designed to be fail-fast (i.e., an exception is thrown when an attempt is made to use an iterator and a concurrent modification of its underlying container is detected). Fail-fast iterators are implemented by keeping track of the "version" of the container that an iterator is associated with, and incrementing a container's version number upon each modification. This "bookkeeping code" is executed, and space for its data is reserved, regardless of the fact whether or not iterators are used. For clients that do not use iterators, a customized container without iteration support can improve performance.

A third common case of unnecessary overhead occurs when single-threaded applications use library classes that are designed with multi-threaded clients in mind. For example, many Java programs frequently concatenate strings via calls to the synchronized method java. lang.StringBuffer.append ( $)^{3}$. This means that a lock must be acquired for each call to this method, which is unnecessary for single-threaded applications. Performance can improved in such cases by rewriting the application to use custom, unsynchronized StringBuffers.

We present a fully automated approach for generating and using customized versions of Java library classes. This approach consists of 5 steps.

1. First, type constraints are used to determine where library classes can be replaced with custom versions without affecting type correctness or program behavior. The result is a set of allocation site candidates that may allocate custom type objects instead of standard library type objects.

2. Static analysis is then used to determine those candidates for which unused library functionality and synchronization can be removed safely from the allocated types.

3. In addition, profile information is collected about the usage characteristics of the customization candidates to determine where the allocation of custom library classes is likely to be profitable. In our whole approach, selecting the training inputs and executing the instrumented program is the only manual step.

4. Based on the static analysis results and the profiling information, custom library classes are automatically generated from a template.

5. Finally, the bytecode of the client application is rewritten to use the generated custom classes. This bytecode rewriting is completely transparent to the programmer.

We applied this approach to a set of benchmark Java applications, and customized various container classes in java.util, as well as class StringBuffer.

\footnotetext{
${ }^{3}$ Java compilers translate uses of the +-operator on String objects into calls to StringBuffer. append ().
} 
We measured speedups ranging from -5 to $78 \%$ (19-24\% on average, depending on the $\mathrm{VM}$ ) and memory footprint reductions ranging from -1 to $46 \%$ (averaging $12 \%$ ). Moreover, the addition of custom classes to the benchmarks resulted in only a modest increase in application size: less than $12 \%$ for all but the smallest programs.

The remainder of this paper is structured as follows. Section 2 presents a motivating example. Sections 3 and 4 present type constraints and their use for determining where custom classes may be used, respectively. Section 5 discusses the steps involved in profiling and generating custom classes. In Section 6, an evaluation of our techniques on a set of benchmarks is presented. Sections 7 and 8 present related work, and conclusions and future work, respectively.

\section{Motivating Example}

We will use an example program that creates several Hashtable objects to illustrate the issues that arise when replacing references to standard library classes with references to custom classes. Class Hashtable is well-suited to serve as a motivating example because: (i) it is part of a complex class hierarchy in which it has both supertypes (e.g., Map and Dictionary) and subtypes (Properties), (ii) several orthogonal optimizations can be applied when creating custom hashtables $^{4}$, and (iii) Hashtables are heavily used by many (legacy) Java applications.

In order to create a customized version of, say, a Hashtable, one could simply create a class CustomHashtable that extends Hashtable and overrides some of its methods. Unfortunately, this approach has significant limitations. In particular, fully lazy allocation is impossible because Hashtable's constructors always allocate certain auxiliary datastructures (e.g., an array of hash-buckets) ${ }^{5}$, and each constructor of CustomHashtable must invoke one of Hashtable's constructors. Moreover, each CustomHashtable object contains all of Hashtable's instance fields, which introduces unneccessary overhead if these fields are unused (as was the case in the example discussed above of redundant iteratorrelated bookkeeping code). Therefore, customized classes will be introduced in a separate branch of the class hierarchy, as is indicated in Figure 1. The figure shows the standard library types Hashtable, Map, Dictionary, and Properties, and the inheritance relationships between them. Also shown are custom container classes CachingHashtable and LazyAllocHashtable, and an abstract class AbstractCustomHashtable that serves as a common superclass of customized versions of Hashtable.

\footnotetext{
${ }^{4}$ One particular optimization that can be applied to custom versions of class Hashtable is the removal of synchronization in cases where the client application is single-threaded. To avoid overhead in such cases, modern Java applications tend to use the similar class HashMap, in which the methods are not synchronized.

${ }^{5}$ While it is possible to specify the initial size of this array of hash-buckets upon construction of a Hashtable-object, the construction of this array-object cannot be avoided altogether if CustomHashtable is a subclass of Hashtable.
} 
We will use the example program shown in Figure 2 to illustrate the issues that arise when introducing custom classes such as those shown in Figure 1. This program creates a number of container objects, and performs some method calls on these objects. Observe that the program contains three allocation sites of type Hashtable and one of type String that we will refer as H1, H2, H3, and $\mathrm{S} 1$, as is indicated in Figure 2 using comments. We will now examine a number of issues that arise when updating allocation sites to refer to custom types.

Calls to methods in external classes. Allocation site $\mathrm{H} 1$ cannot be updated to refer to a custom type because the object allocated at $\mathrm{H} 1$ is passed to a constructor of javax.swing. JTree that expects an argument of type java.util. Hashtable. Since the code in class JTree is not under our control, the type of the parameter of JTree's constructor must remain java.util.Hashtable, which implies that the types of h1 and H1 must remain java.util.Hashtable as well. Similar issues arise for calls to library methods whose return type is a concrete ${ }^{6}$ standard container, such as the call to System.getProperties() on line 11, which returns an object of type java.util.Properties, a subtype of java.util.Hashtable.

Preserving type-correctness. Allocation sites H2 and H3 may be updated to refer to, for example, type CachingHashtable. However, if we make this change, we must also update h2 and h3 to refer to a superclass of CachingHashtable (i.e., CachingHashtable, AbstractCustomHashtable, Map, Dictionary, or Object) because the assignments on lines 5 and 6 would otherwise not be type-correct. The method calls h2.put ("FOO", "BAR") and h2. putAll (c) impose the additional requirement that the put() and putAll() methods must be visible in the type of h2, and hence that h2's type must be CachingHashtable, AbstractCustomHashtable, or Map. Furthermore, the assignment $\mathrm{h} 2=\mathrm{h} 3$ is only type-correct if the type of $\mathrm{h} 2$ is the same as or a supertype of the type of $\mathrm{h} 3$, and the assignments Properties $\mathrm{p} 1=$ System.getProperties () and $\mathrm{h} 2=\mathrm{p} 1$ require that the type of $\mathrm{h} 2$ must be a supertype of java.util.Properties. Combining all of these requirements, we infer that allocation sites $\mathrm{H} 2$ and $\mathrm{H} 3$ can only be updated to allocate CachingHashtable objects if both h2 and h3 are declared to be of type Map.

Preserving the behavior of casts. The cast expression on line 16 (indicated as C1) presents another interesting case. Observe that only objects allocated at sites H3 and S1 may be bound to parameter 0 . In the transformed program, the cast expression must succeed and fail in exactly the same cases as before. In this case, if the type of the object allocated at site H3 is changed to CachingHashtable, changing the type of the cast to, for example, AbstractCustomHashtable will preserve the behavior of the cast (it will still succeed when parameter o points to an object allocated at site $\mathrm{H} 3$ and it will

\footnotetext{
6 The use of concrete container types such as Hashtable (as opposed to abstract container types such as Map) in the signature of public library methods is often an indication of poor design, because it unnecessarily exposes implementation details. Nonetheless, this practice is pervasive. We counted 165 public methods in the JDK 1.3.1 standard libraries whose signature refers to Hashtable, Vector, or Properties.
} 
still fail when o points to an object allocated at site S1). Furthermore, the assignment Hashtable $\mathrm{h} 4=$ (Hashtable) 0 is only type-correct if the type of $\mathrm{h} 4$ is a supertype of the type referenced in the cast expression, and the method call h4.contains $(\ldots)$ implies that h4's type must define the contains $(\ldots)$ method (in other words, h4 must have type AbstractCustomHashtable or a subtype thereof). We conclude from the above discussion that having the cast refer to type AbstractCustomHashtable and declaring h4 to be of type AbstractCustomHashtable is a valid solution ${ }^{7}$.

The lessons learned from the above example can be summarized as follows: The customized program must satisfy interface-compatibility constraints that are due to the exchange of standard container objects with third-party libraries and type-correctness constraints implied by program constructs such as assignments that constrain the types of their subexpressions. Moreover, run-time behavior must be preserved for casts and instanceof operations.

Lines 5b, 15b, and 16b in Figure 2 indicate how allocation sites, declarations, and cast expressions in the original example program have been replaced with references to custom classes in accordance with the requirements that we discussed informally in this section. We will now turn our attention to a more precise treatment of these requirements.

\section{Type Constraints}

To determine where the types of allocation sites and declarations can be updated to refer to custom types in a way that preserves the program's type-correctness and behavior, we will use an existing framework of type constraints [18,25]. For each program construct, one or more type constraints express the subtyperelationships that must exist between the declared types of the construct's constituent expressions, in order for that program construct to be type-correct. By definition, a program is type-correct if the type constraints for all constructs in that program are satisfied.

Unlike previous work on refactoring and replacing types in a program [25], our rewriting involves more than moving declared types up or down in an existing or extended class hierarchy. Instead we will move type declarations more or less horizontally: standard types in some position of the standard library hierarchy are replaced by custom types in a corresponding position in a newly added branch of the hierarchy. Sometimes vertical motion of declared types will still be useful however, because fields or variables can only hold references to both standard types and customized types if they are declared a supertype of both the standard classes and the custom classes.

In this section, we will therefore first introduce the existing constraint framework that was used in, e.g., [25]. This framework is then extended in Section 4 to accommodate the horizontal type replacements.

\footnotetext{
${ }^{7}$ Several other solutions exist. For example, variable $\mathrm{h} 4$ and cast $\mathrm{C} 1$ can both receive type CachingHashtable.
} 


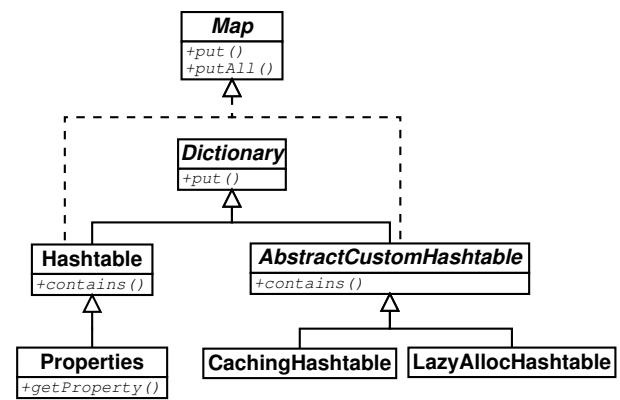

Fig. 1. A fragment of the standard container hierarchy in package java.util.*, augmented with several customized containers. The figure shows, for a number of relevant methods, the most general type in which they are declared.

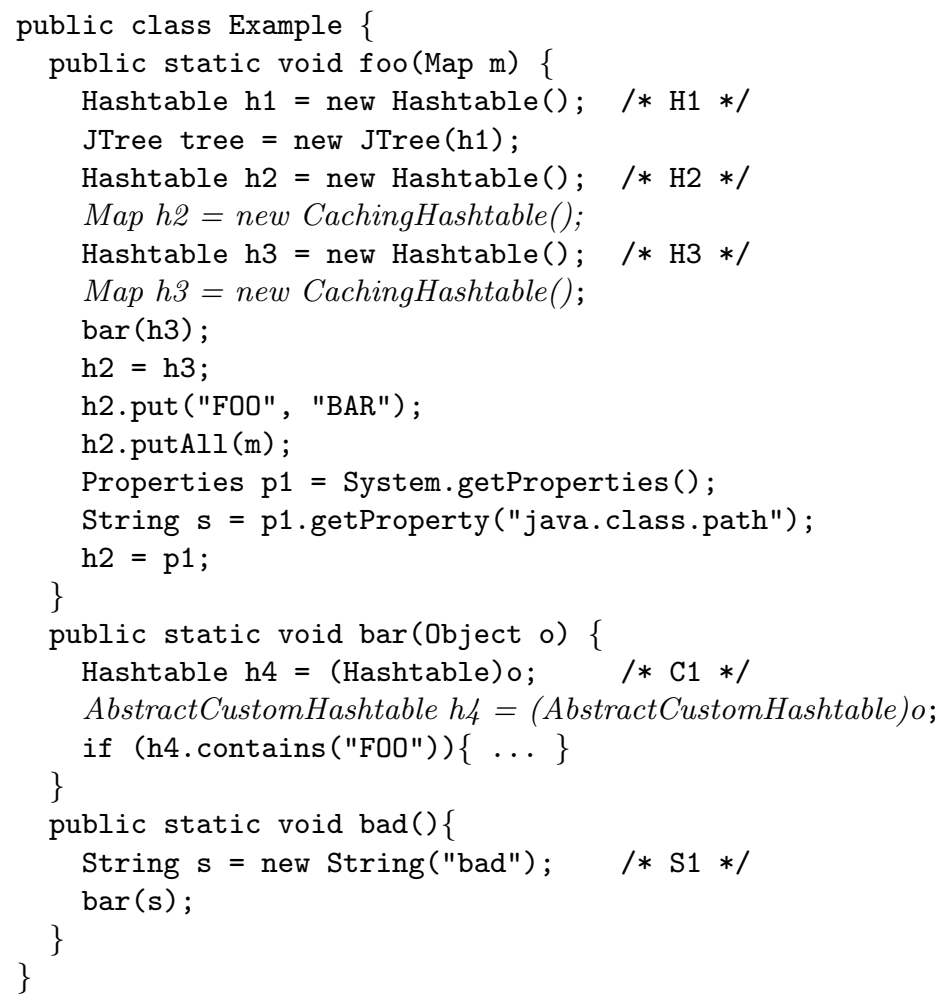

Fig. 2. This figure shows an example program $P_{1}$, which consists of lines 1-23 excluding lines $5 \mathrm{~b}, 6 \mathrm{~b}$, and $16 \mathrm{~b}$. A customized version of this program that uses the custom class hierarchy of Figure 1 can be obtained from $P_{1}$ by replacing lines 5,6 , and 16 , with lines $5 \mathrm{~b}, 6 \mathrm{~b}$, and $16 \mathrm{~b}$, respectively. 
In the remainder of this paper, we assume that the original program is typecorrect. Moreover, we assume that the original program does not contain any up-casts (i.e., casts $(C) E$ in which the type of expression $E$ is a subclass of $C$ ). This latter assumption is not a restriction, as there is no need for up-casts in Java byte $\operatorname{code}^{8}$.

\subsection{Notation and Terminology}

Following [25], we use the term declaration element to refer to declarations of local variables, parameters in static, instance, and constructor methods, fields, and method return types, and to type references in cast expressions. In what follows, $v, v^{\prime}$ denote variables, $M, M^{\prime}$ denote methods, $F, F^{\prime}$ denote fields, $C, C^{\prime}$ denote classes, $I, I^{\prime}$ denote interfaces, and $T, T^{\prime}$ denote types ${ }^{9}$. It is important to note that the symbol $M$ denotes a method together with all its signature and return type information and the reference to its declaring type. Similarly, $F$ and $C$ denote a field and a type, respectively, together with its name, type in which it is declared and, in the case of fields, its declared type.

Moreover, the notation $E, E^{\prime}$ will be used to denote an expression or declaration element at a specific point in the program, corresponding to a specific node in the program's abstract syntax tree. We will assume that type information about expressions and declaration elements is available from the compiler.

A method $M$ is virtual if $M$ is not a constructor, $M$ is not private and $M$ is not static. Definition 1 defines the concept of overriding ${ }^{10}$ for virtual methods.

Definition 1 (overriding). A virtual method $M$ in type $C$ overrides a virtual method $M^{\prime}$ in type $B$ if $M$ and $M^{\prime}$ have identical signatures and $C$ is equal to $B$ or $C$ is a subtype of $B .^{11}$ In this case, we also say that $M^{\prime}$ is overridden by $M$.

Definition 2 defines, for a given method $M$, the set RootDefs $(M)$ of methods $M^{\prime}$ that are overridden by $M$ that do not override any methods except for themselves. Since we assume the original program to be type-correct, this set is guaranteed to be non-empty. For example, in the standard collection hierarchy, we have that RootDefs(Hashtable.put ()) $=\{$ Map.put (), Dictionary $\cdot \operatorname{put}()\}$ because Map and Dictionary are the most general types that declare put() methods that are overridden by Hashtable.put().

Definition 2 (RootDefs). Let $M$ be a method. Define:

$$
\operatorname{RootDefs}(M)=\left\{\begin{array}{c}
M^{\prime} \mid M \text { overrides } M^{\prime}, \text { and there exists no } M^{\prime \prime}\left(M^{\prime \prime} \neq M^{\prime}\right) \\
\text { such that } \left.M^{\prime} \text { overrides } M^{\prime \prime}\right\}
\end{array}\right.
$$

${ }^{8}$ In Java source code, up-casts are sometimes needed for explicit resolution of overloaded methods.

${ }^{9}$ In this paper, the term type will denote a class or an interface.

${ }^{10}$ Note that, according to Definition 1, a virtual method overrides itself.

11 This definition of overriding does not take into account issues related to access rights and throws-clauses. A precise definition of overriding that takes these issues into account is a topic for future work. 
Figure 3 shows the notation used to express type constraints. A constraint variable $\alpha$ is one of the following: $T$ (a type constant), $[E]$ (representing the type of an expression or declaration element $E$ ), $\operatorname{Decl}(M)$ (representing the type in which method $M$ is declared), or $\operatorname{Decl}(F)$ (representing the type in which field $F$ is declared). A type constraint is a relationship between two or more constraint variables that must hold in order for a program to be type-correct. In this paper, a type constraint has one of the following forms: (i) $\alpha_{1} \triangleq \alpha_{2}$, indicating that $\alpha_{1}$ is defined to be the same as $\alpha_{2}$ (ii) $\alpha_{1} \leq \alpha_{2}$, indicating that $\alpha_{1}$ must be equal to or be a subtype of $\alpha_{2}$, (iii) $\alpha_{1}=\alpha_{2}$, indicating that $\alpha_{1} \leq \alpha_{2}$ and $\alpha_{2} \leq \alpha_{1}$, (iv) $\alpha_{1}<\alpha_{2}$, indicating that $\alpha_{1} \leq \alpha_{2}$ but not $\alpha_{2} \leq \alpha_{1}$, (v) $\alpha_{1}^{L} \leq \alpha_{1}^{R}$ or $\cdots$ or $\alpha_{k}^{L} \leq \alpha_{k}^{R}$, indicating that $\alpha_{j}^{L} \leq \alpha_{j}^{R}$ must hold for at least one $j, 1 \leq j \leq k$, (vi) $\alpha_{1} \not \leq \alpha_{2}$, indicating that $\alpha_{1}$ must not be equal to or be a subtype of $\alpha_{2}$

In discussions about types and subtype-relationships that occur in a specific program $P$, we will use the notation of Figure 3 with subscript $P$. For example, $[E]_{P}$ denotes the type of expression $E$ in program $P$, and $T^{\prime} \leq_{P} T$ denotes a subtype-relationship that occurs in program $P$. In cases where the program under consideration is unambiguous, we will frequently omit these $P$-subscripts.

\subsection{Inferring Type Constraints}

We will now present the rules that will be used for inferring type constraints from various Java constructs and analysis facts.

Type-Correctness Constraints Rules (1)-(17) in Figure 4 shows the type constraints that are implied by a number of common Java program constructs. For example, constraint (1) states that an assignment $E_{1}=E_{2}$ is type correct if the type of $E_{2}$ is the same as or a subtype of the type of $E_{1}$. For a virtual method call $E . m\left(E_{1}, \cdots, E_{n}\right)$ that statically resolves to a method $M$, we define the type of the call-expression to be the same as $M$ 's return type (rule (2)), and we require that the type of each actual parameter $E_{i}$ must be the same as or a subtype of the type of the corresponding formal parameter $\operatorname{Param}(M, i)$ (rule (3)). Moreover, a declaration of a method with the same signature as $M$ must occur in a supertype of the type of $E$. This latter fact is expressed in rule (4) using Definition 2 by way of an or-constraint. For cast expressions of the form $(C) E$, rule (21) defines the type of the cast to be $C$. Moreover, rule (12) states the requirement that the type of $E$ must be a supertype of $C^{12}$.

Rules (18)-(22) define the types of variables, parameters, fields, method return types, casts, and allocation sites in the original program.

Behavior-Preserving Constraints The type constraints discussed thus far are only concerned with type-correctness. In general, additional constraints must

\footnotetext{
${ }^{12}$ In [25], this constraint for cast-expressions reads $[E] \leq[(C) E] \operatorname{or}[(C) E] \leq E$. We can use a simplified version here because we make the assumption that the original program does not contain up-casts.
} 
$[E] \quad$ the type of expression or declaration element $E$

$[M] \quad$ the declared return type of method $M$

$[F] \quad$ the declared type of field $F$

$\operatorname{Decl}(M) \quad$ the type that contains method $M$

$\operatorname{Decl}(F) \quad$ the type that contains field $F$

$\operatorname{Param}(M, i)$ the $i$-th formal parameter of method $M$

$T^{\prime} \leq T \quad T^{\prime}$ is equal to $T$, or $T^{\prime}$ is a subtype of $T$

$T^{\prime}<T \quad T^{\prime}$ is a proper subtype of $T$ (i.e., $T^{\prime} \leq T$ and not $T \leq T^{\prime}$ )

Fig. 3. Type constraint notation.

\begin{tabular}{|c|c|c|}
\hline program construct(s)/analysis fact(s) & implied type constraint(s) & \\
\hline $\begin{array}{c}\text { assignment } \quad E_{1}=E_{2} \\
\end{array}$ & $\begin{array}{c}{\left[E_{2}\right] \leq\left[E_{1}\right]} \\
\end{array}$ & $(1)$ \\
\hline $\begin{array}{l}\text { method call } \\
\text { E.m }\left(E_{1}, \cdots, E_{n}\right) \\
\text { to a virtual method } M\end{array}$ & $\begin{array}{c}{\left[E . m\left(E_{1}, \cdots, E_{n}\right)\right] \triangleq[M]} \\
{\left[E_{i}\right] \leq[\operatorname{Param}(M, i)]} \\
{[E] \leq \operatorname{Decl}\left(M_{1}\right) \text { or } \cdots \text { or }[E] \leq \operatorname{Decl}\left(M_{k}\right)} \\
\text { where } \operatorname{RootDefs}(M)=\left\{M_{1}, \cdots, M_{k}\right\}\end{array}$ & $\begin{array}{l}(2) \\
(3) \\
(4)\end{array}$ \\
\hline access $E . f$ to field $F$ & $\begin{array}{c}{[E . f] \triangleq[F]} \\
{[E] \leq \operatorname{Decl}(F)}\end{array}$ & $\begin{array}{l}(5) \\
(6) \\
\end{array}$ \\
\hline return $E$ in method $M$ & {$[E] \leq[M]$} & $(7)$ \\
\hline $\begin{array}{c}\text { constructor call new } C\left(E_{1}, \cdots, E_{n}\right) \\
\text { to constructor } M\end{array}$ & {$\left[E_{i}\right] \leq[\operatorname{Param}(M, i)]$} & (8) \\
\hline $\begin{array}{c}\text { direct call } \\
\text { E.m }\left(E_{1}, \cdots, E_{n}\right) \\
\text { to method } M\end{array}$ & $\begin{array}{c}{\left[E \cdot m\left(E_{1}, \cdots, E_{n}\right)\right] \triangleq[M]} \\
{\left[E_{i}\right] \leq[\operatorname{Param}(M, i)]} \\
{[E] \leq \operatorname{Decl}(M)}\end{array}$ & $\begin{array}{r}(9) \\
(10) \\
(11)\end{array}$ \\
\hline $\begin{array}{l}\text { cast } \\
(C) E\end{array}$ & $\begin{array}{c}{[(C) E] \leq[E]} \\
\text { if }[E] \text { is a class }\end{array}$ & $(12)$ \\
\hline for every type $T$ & $\begin{array}{c}T \leq \text { java.lang.Object } \\
{[\text { null] } \leq \mathrm{T}}\end{array}$ & $\begin{array}{l}(13) \\
(14)\end{array}$ \\
\hline implicit declaration of this in method $M$ & {$[$ this $] \triangleq \operatorname{Decl}(M)$} & $(15)$ \\
\hline declaration of method $M($ declared in type $T)$ & $\operatorname{Decl}(M) \triangleq T$ & $(16)$ \\
\hline declaration of field $F($ declared in type $T)$ & $\operatorname{Decl}(F) \triangleq T$ & $(17)$ \\
\hline $\begin{array}{l}\text { explicit declaration } \\
\text { of variable or method parameter } T v\end{array}$ & {$[v] \triangleq T$} & $(18)$ \\
\hline declaration of method $M$ with return type $T$ & {$[M] \triangleq T$} & $(19)$ \\
\hline declaration of field $F$ with type $\quad T$ & {$[F] \triangleq T$} & $(20)$ \\
\hline cast $(T) E$ & {$[(T) E] \triangleq T$} & $(21)$ \\
\hline expression new $C\left(E_{1}, \cdots, E_{n}\right)$ & {$\left[\right.$ new $\left.C\left(E_{1}, \cdots, E_{n}\right)\right] \triangleq C$} & $(22)$ \\
\hline $\begin{array}{c}M^{\prime} \text { overrides } M, \\
M^{\prime} \neq M \\
\end{array}$ & $\begin{aligned}\left.\operatorname{Param}\left(M^{\prime}, i\right)\right] & =[\operatorname{Param}(M, i)] \\
{\left[M^{\prime}\right] } & =[M]\end{aligned}$ & $\begin{array}{l}(23) \\
(24) \\
\end{array}$ \\
\hline $\begin{array}{l}\text { for each cast expression }(C) E, \text { and each } \\
\text { allocation expression } E^{\prime} \in \operatorname{Points} T o(P, E) \\
\text { such that }\left[E^{\prime}\right]_{P} \leq[(C) E]_{P}\end{array}$ & {$\left[E^{\prime}\right] \leq[(C) E]$} & $(25)$ \\
\hline $\begin{array}{l}\text { for each cast expression }(C) E \text {, and each } \\
\text { allocation expression } E^{\prime} \in \operatorname{Points} T o(P, E) \\
\text { such that }\left[E^{\prime}\right]_{P} \not \subset[(C) E]_{P}\end{array}$ & {$\left[E^{\prime}\right] \not \subset[(C) E]$} & $(26)$ \\
\hline $\begin{array}{l}\text { expression } E \text { that occurs in the libraries } \\
\text { such that }[E]_{P}=T\end{array}$ & {$[E]=T$} & (27) \\
\hline
\end{tabular}

Fig. 4. Type constraints for a set of core Java language features. Rules (1)-(17) define the types of expressions and impose constraints between the types of expressions and declaration elements. Rules (18)-(22) define the types of declaration elements and allocation expressions in the original program. Rules (23)-(26) show additional type constraints that are needed for the preservation of program semantics. Rule (27) shows an additional type constraint needed to preserve interface-compatibility. 


$$
\begin{array}{ll}
\text { PointsTo }\left(P_{1}, \mathrm{~h} 1\right)=\{\text { H1 }\} & \text { PointsTo }\left(P_{1}, \mathrm{o}\right)=\{\mathrm{H} 3, \mathrm{~S} 1\} \\
\text { PointsTo }\left(P_{1}, \mathrm{~h} 2\right)=\{\mathrm{H} 2, \mathrm{H} 3, \mathrm{P} 1\} & \text { PointsTo }\left(P_{1}, \mathrm{p}\right)=\{\mathrm{P} 1\} \\
\text { PointsTo }\left(P_{1}, \mathrm{~h} 3\right)=\{\text { H3 }\} & \text { PointsTo }\left(P_{1}, \mathrm{~s}\right)=\{\mathrm{S} 1\} \\
\text { PointsTo }\left(P_{1}, \mathrm{~h} 4\right)=\{\text { H3 }\} &
\end{array}
$$

Fig. 5. Points-to information for program $P_{1}$ of Figure 2 , as computed using a variation on the flow-insensitive, context-insensitive 0-CFA algorithm [17] that propagates allocation sites rather than types. Here, P1 represents the allocation site(s) at which the Properties objects returned by System.getProperties() are allocated.

be imposed to ensure that program behavior is preserved. Rules (23) and (24) state that overriding relationships that occur in the original program $P$ must also occur in the rewritten program $P^{\prime}$.

Rules (25) and (26) state that the execution behavior of a cast $(C) E$ must be preserved. Here, the notation $\operatorname{Points} T o(P, E)$ refers to be the set of objects (identified by their allocation sites) that an expression $E$ in program $P$ may point to. Any of several existing algorithms [20,13,21] can be used to compute this information. Figure 5 shows the points-to information that will be used in the examples below. Rule (25) ensures that for each $E^{\prime}$ in the points-to set of $E$ for which the cast succeeds, the cast will still succeed in $P^{\prime}$. Likewise, Rule (26) enforces that for each $E^{\prime}$ in the points-to set of $E$ for which the cast fails, the cast will still fail in $P^{\prime}$.

Interface-Compatibility Constraints Finally, we need to ensure that the customized program preserves interface-compatibility. To this end, we impose the additional constraint of rule (27) in Figure 4. This rules states that the types of declarations and expressions that occur in external class libraries cannot be changed.

\section{Introducing Custom Classes}

The introduction of custom classes proceeds using the following steps. First, the class hierarchy is extended with custom classes and auxiliary types. Then, type constraints are computed for the program with respect to this extended class hierarchy. This is followed by a step in which all constraints are transformed into simple equality or subtype constraints. Finally, the constraint system is solved in order to determine where custom classes can be used.

\subsection{Extension of the Class Hierarchy}

Before computing type constraints, we extend the class hierarchy with the custom classes that we would like to introduce. Adding these types prior to the construction of the constraints will allow us to determine where custom container classes may be introduced. In addition to the custom classes themselves, some auxiliary types will be added to the hierarchy. In what follows, we use the term customizable class to refer to a class for which we would like to introduce a custom version. Specifically, we extend the original class hierarchy as follows: 
- For each customizable class $C$ with superclass $B$, a class $C$ ustom $C$ is created that contains methods and fields that are identical to those in $C$. If $B$ is not customizable, then Custom C's superclass is B, otherwise it is CustomB.

- For each customizable class $C$, a type $C^{\top}$ is introduced, and both $C$ and Custom $C$ are made a subtype of $C^{\top}$. Type $C^{\top}$ contains declarations of all methods in $C$ that are not declared in any superclass of $C$.

- For each customizable container $C$, a type $C^{\perp}$ is introduced, and $C^{\perp}$ is made a subclass of both $C$ and $C$ ustom $C$. Type $C^{\perp}$ contains no methods.

Multiple inheritance is used because it allows us to express that the type of an allocation site $E$ should be either $C$ or $C$ ustom $C$ by way of subtype-constraints $[E] \leq C^{\top}$ and $C^{\perp} \leq[E]$. It is important to note that these multiple inheritance relations are only used during the solving of the type constraints and that the customized program does not refer to any type $C^{\top}$ or $C^{\perp}$.

Figure 6 shows the parts of the class hierarchy relevant for the customization of the example program of Figure 2 after adding the classes CustomHashtable and CustomProperties, and the additional types Hashtable ${ }^{\top}, \mathrm{Hashtable}^{\perp}$, Properties $^{\top}$ and Properties ${ }^{\perp}$. Section 4.4 describes how the CustomC classes can be further transformed, and turned into a separate class hierarchy such as the one shown earlier in Figure 1.

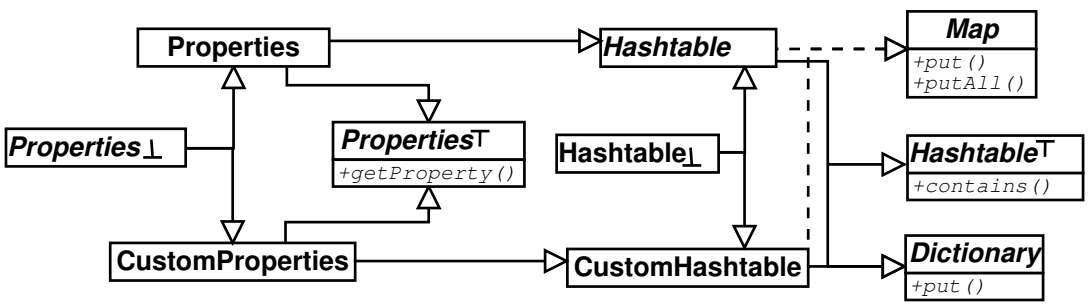

Fig. 6. Fragment of the collection hierarchy in java.util.* after extending it with custom classes and interfaces. For each class/interface, one or more of the methods defined/declared in that class/interface are shown.

The original program always allocates an object of type $C$ at an allocation site new $C\left(E_{1}, \cdots, E_{n}\right)$, as was reflected by rule (22) in Figure 4 . In the transformed program, we want to allow solutions where the allocated object is either of type $C$ or of type CustomC. To this end, we replace rule (22) with rules (22)(a)-(22)(c) shown in Figure 7.

Figure 8 shows all non-trivial type constraints for the example program of Figure 2. For convenience, we have annotated each constraint in Figure 8 with the line number(s) in the source code from which it was derived, and with the number of the rule(s) in Figure 4 responsible for the constraint's creation. 


\begin{tabular}{|c|cr|}
\hline program construct(s)/analysis facts & implied type constraint(s) & \\
\hline \hline expression new $C\left(E_{1}, \cdots, E_{n}\right)$ & {$\left[\right.$ new $\left.C\left(E_{1}, \cdots, E_{n}\right)\right] \leq C^{\top}$} & $(22)(\mathrm{a})$ \\
$C$ is a customizable class & $C^{\perp} \leq\left[\right.$ new $\left.C\left(E_{1}, \cdots, E_{n}\right)\right]$ & $(22)(\mathrm{b})$ \\
\hline expression new $C\left(E_{1}, \cdots, E_{n}\right)$ & {$\left[\right.$ new $\left.C\left(E_{1}, \cdots, E_{n}\right)\right] \triangleq C$} & $(22)(\mathrm{c})$ \\
$C$ is a non-customizable class & & \\
\hline
\end{tabular}

Fig. 7. Revised type constraint rules for allocation sites and casts.

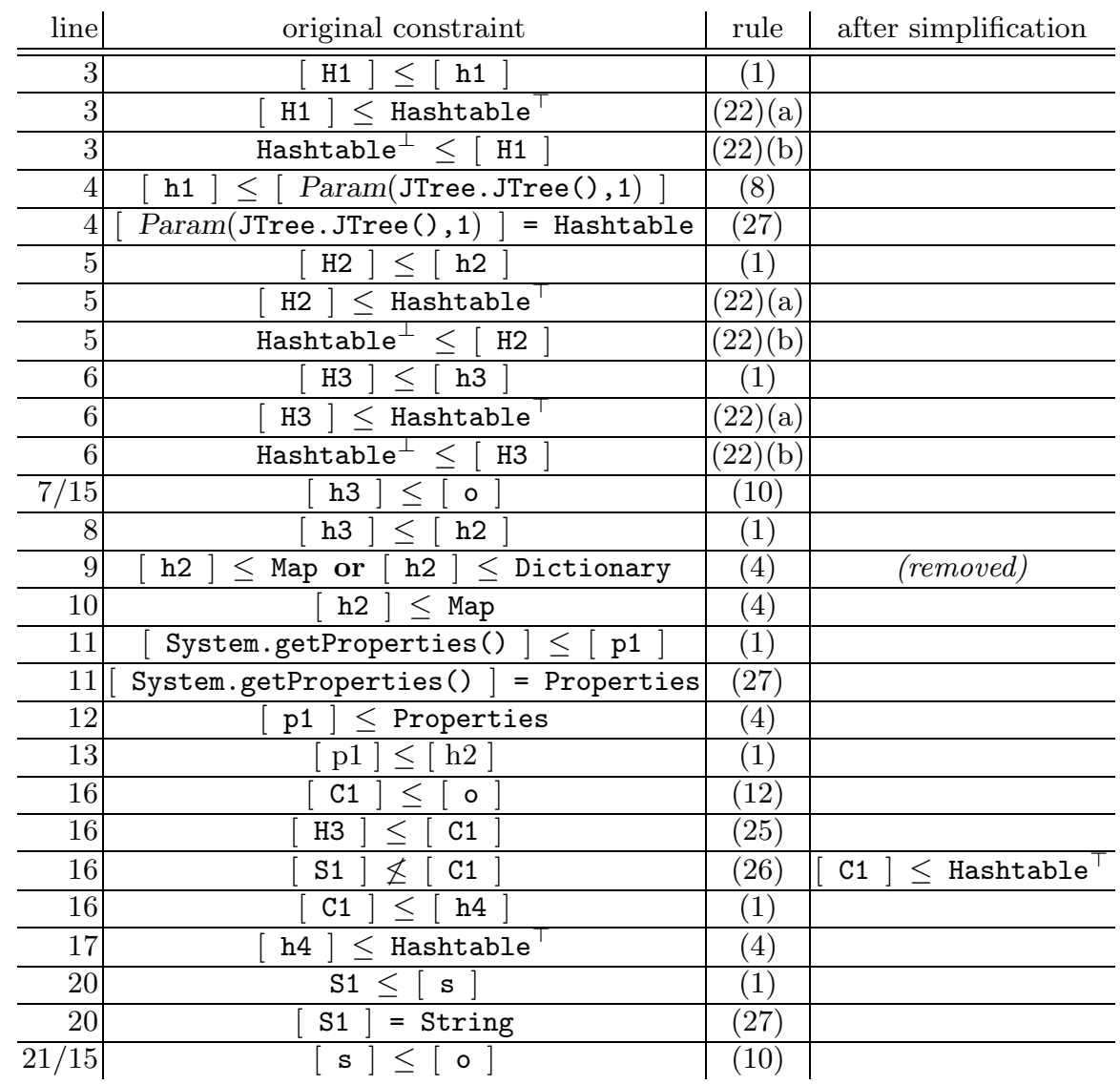

Fig. 8. Type constraints for the example program of Figure 2(a) as derived according to Figure 4. The rows in the table show, from left to right, the line in the source code from which the constraint was derived, the constraint itself, the rule that triggered the creation of the constraint, and the constraint after simplification (where applicable). 


\subsection{Constraint Simplification}

Constraints of the form $[E] \leq C_{1}$ or $\cdots$ or $[E] \leq C_{k}$ (generated using rule (4)) give rise to bifurcations when traversing the solution space, and constraints of the form $\left[E^{\prime}\right] \not \subset[(C) E]$ (generated using rule (26)) make it impossible to define a monotone iteration step. Therefore, in order to simplify the process of constraint solving, we first perform a step in which all constraints are reduced to the simple forms $[x] \leq[y],[x]=[y]$, and $[x] \triangleq[y]$.

Simplification of Disjunctions A virtual method call E.m $\left(E_{1}, \cdots, E_{n}\right)$ to a method $M$ gives rise to a disjunction $[E] \leq C_{1}$ or $\cdots$ or $[E] \leq C_{k}$ if $[E]$ has multiple supertypes $C_{1}, \cdots, C_{k}$ in which method $m(\cdots)$ is declared such that there is not a single method $M$ that is overridden by all $C_{i} \cdot m(\cdots)$, for all $i, 1 \leq i \leq k$. Our approach will be to replace the entire disjunction by one of its branches $[E] \leq C_{j}$, for some $j, 1 \leq j \leq k$. Note that by imposing a stronger constraint on $[E]$, we are potentially reducing the number of solutions of the constraint system. Nevertheless, at least one solution is guaranteed to exist: The original program fulfills each of the components of the original disjunction ${ }^{13}$, so it will meet the simplified constraint as well.

Still, choosing the branch to replace the disjunction requires some consideration. Consider the constraint: [ h2 ] $\leq$ Map or [ h2 ] $\leq$ Dictionary that was generated due to the call h2.put ("FOO", "BAR") in the example program of Figure 2. If we simplify this constraint to: [ h2 ] $\leq$ Dictionary, we obtain a constraint system in which variable $\mathrm{h} 2$ must be a subtype of both Map and Dictionary, as well as a supertype of java.util.Properties. This implies that h2's type must be a subtype java.util. Hashtable, which, in turn, requires that allocation sites H2 and H3 must remain of type java. util. Hashtable, preventing us from customizing these allocation sites. On the other hand, replacing the original disjunction with: [ h2 ] $\leq$ Map allows us to infer the solution shown earlier in Figure 2(b), in which allocation sites H2 and H3 have been customized. Clearly, some choices for simplifying disjunctions are better than others.

We use the following approach for the simplification of disjunctions. First, any constraint $[x] \leq C_{1}$ or $\cdots$ or $[x] \leq C_{n}$ for which there already exists another constraint $[x] \leq C_{j}$ can simply be removed by subsumption, as the latter constraint implies the former. Second, we use the heuristic that any constraint $[x] \leq C_{1}$ or $\cdots$ or $[x] \leq C_{n}$, for which there exists another constraint $[y] \leq C_{j}$, for some unique $j(1 \leq j \leq n)$ such that $\operatorname{PointsTo}(P, x) \cap \operatorname{PointsTo}(P, y) \neq \emptyset$. is simplified to $[x] \leq C_{j}$. If no constraint $[y] \leq C_{j}$ exists, the disjunction is simplified by making an arbitrary choice. The results of this approach have been satisfactory so far. If the loss of precision becomes a problem, one could compute the results obtained for all possible choices for each disjunction, and select a maximal solution.

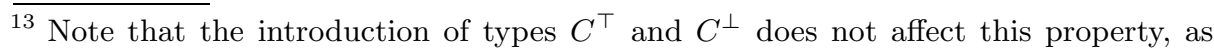
they do not give rise to additional disjunctions. 
Simplification of $\not$-Constraints Constraints of the form $\left[E^{\prime}\right] \not \subset[(C) E]$ are introduced by rule (25) in order to preserve the behavior of casts that may fail. For example, in the program of Figure 2 the cast on line 14 fails when method $\operatorname{bar}()$ is called from method bad(), because in this case o will point to a String-object that was allocated at allocation site S1.

Our approach will be to introduce additional constraints that are sufficient to imply the $\not{Z}$-constraint. Specifically, for each cast $(C) E$ for which the points-to set PointsTo $(P, E)$ contains an expression $E^{\prime}$ such that $\left[E^{\prime}\right]_{P} \not C$, we introduce a constraint $[(C) E] \leq C^{\top}$. This additional constraint prevents the generalization of the target type of a cast in situations where that would change a failing cast into a succeeding cast.

It is easy to see that the addition of this constraint ensures that the behavior of failing casts is preserved in the customized program $P^{\prime}$. Suppose that $(C) E$ is a cast that may fail in the original program $P$. Then, there exists an $E^{\prime} \in \operatorname{Points} T o(P, E)$ for which $\left[E^{\prime}\right]_{P} \not C$. Since $P$ does not instantiate any custom classes, we also know that $\left[E^{\prime}\right]_{P} \not \subset$ Custom $C$, and therefore that $\left[E^{\prime}\right]_{P} \not \subset C^{\top}$. Hence, requiring that $[(C) E] \leq C^{\top}$ ensures that the constraint $\left[E^{\prime}\right] \not \subset[(C) E]$ is satisfied in $P^{\prime}$.

Example. Figure 8 shows the simplified type constraints for the program of Figure 2. For the disjunction [ h2 ] $\leq$ Map or [ h2 ] $\leq$ Dictionary in Figure 8, there already exists another, stronger constraint [ h2 ] $\leq$ Map, so it can simply be removed. Furthermore, the $\not \subset$-constraint [ S1 ] $\not \leq[\mathrm{C} 1]$ is replaced with a constraint $[\mathrm{C} 1] \leq$ Hashtable $^{\top}$.

\subsection{Solving the Constraints}

Now that all constraints are of the forms $E \leq E^{\prime}, E=E^{\prime}$, and $E \triangleq E^{\prime}$ solving the constraint system is straightforward. First, we create a set of equivalence classes of declaration elements and expressions that must have exactly the same type, and we extend the $\leq$ relationship to equivalence classes in the obvious manner. Then, we compute the set of possible types for each equivalence class using an optimistic algorithm. This algorithm associates a set $S_{E}$ of types with each equivalence class $E$, which is initialized as follows:

- For each equivalence class $E$ that contains an allocation expression $E \equiv$ new $C, S_{E}$ is initialized to contain the types $C$ and CustomC.

- For each equivalence class $E$ that does not contain any allocation expressions, $S_{E}$ is initialized to contain all types except $C^{\top}$ and $C^{\perp}$, for all $C$.

Then, in the iterative phase of the algorithm, the following steps are performed repeatedly until a fixpoint is reached:

- For each pair of equivalence classes $D, E$ such that there exists a type constraint $D \leq E$, we remove from $S_{D}$ any type that is not a subtype of a type that occurs in $S_{E}$.

- For each pair of equivalence classes $D, E$ such that there exists a type constraint $D \leq E$, we remove from $S_{E}$ any type that is not a supertype of a type that occurs in $S_{D}$. 
Termination of this algorithm is ensured because each iteration decreases the number of elements in at least one set, and there is a finite number of sets. Moreover, each equivalence class will contain at least the type that is associated with its elements in the original program.

\begin{tabular}{c|c} 
equivalence class & possible types \\
\hline \hline$\{\mathrm{p} 1$, Properties $\}$ & $\{$ Properties $\}$ \\
\hline$\{\mathrm{h} 1\}$ & $\{$ Hashtable $\}$ \\
\hline$\{\mathrm{H} 1\}$ & $\{$ Hashtable $\}$ \\
\hline$\{\mathrm{h} 2\}$ & $\{$ Map, Hashtable, CustomHashtable $\}$ \\
\hline$\{\mathrm{H} 2\}$ & $\{$ Hashtable, CustomHashtable $\}$ \\
\hline$\{\mathrm{h} 3\}$ & $\{$ Map, Hashtable, CustomHashtable $\}$ \\
\hline$\{\mathrm{H} 3\}$ & $\{$ Hashtable, CustomHashtable $\}$ \\
\hline$\{\mathrm{h} 4\}$ & $\{$ Hashtable, CustomHashtable $\}$ \\
\hline$\{\mathrm{C} 1\}$ & $\{$ Hashtable, CustomHashtable $\}$ \\
\hline$\{\mathrm{o}\}$ & $\{$ Object $\}$ \\
\hline$\{\mathrm{s}\}$ & $\{$ String $\}$ \\
\hline
\end{tabular}

Fig. 9. Possible types computed for each equivalence class.

Figure 9 shows the sets of types computed for each of the equivalence classes in our example. The interpretation of these sets of types requires some remarks:

- Figure 9 depicts many possible solutions. In each solution, a single type in $S_{E}$ is chosen for each equivalence class $E$.

- If type $T$ occurs in $S_{E}$, then at least one solution to the constraint system exists in which the elements in $E$ have type $T$.

- Selecting types for different equivalence classes can in general not be done independently. For any given pair of equivalence classes $D$ and $E$, choosing an arbitrary element in $S_{D}$ for equivalence class $D$, and an arbitrary element in $S_{E}$ for equivalence class $E$ may result in a type-incorrect program.

- The previous observation particularly applies to two equivalence classes associated with allocation sites $A_{1}$ and $A_{2}$. Selecting type $C$ for (the equivalence class containing) $A_{1}$ may prevent us from selecting type Custom $C$ for (the equivalence class containing) $A_{2}$. For example, if a call bar (h2) is added to method Example.main(), we have the choice of: (i) customizing both $\mathrm{H} 2$ and H3 or (ii) not customizing both $\mathrm{H} 2$ and H3. However, customizing H2 but not H3 (or vice versa) will not preserve the behavior of cast $\mathrm{C} 1$.

- However, we conjecture that a solution exists in which type CustomC is selected for all equivalence classes $E$ such that Custom $C \in S_{E}$.

A more precise treatment of these properties is currently in progress.

\subsection{Pragmatic Issues and Further Customization}

There are several issues that require straightforward extensions to our basic approach. These include the treatment of subtypes of standard library classes 
(e.g., an application declaring a class MyHashtable that extends Hashtable), and limiting the introduction of custom classes in the presence of serialization. Space limitations prevent us from providing more details.

Thus far, we have presented how variables and allocation sites of type $C$ can be updated to refer to type CustomC. At this point it has become easy to replace Custom $C$ with a small hierarchy of custom classes such as the one shown in Figure 1 by applying refactorings [10,25] as follows:

- Split class CustomC into an abstract superclass AbstractCustomC (that only contains abstract methods) and a concrete subclass Custom $C$. Declarations and casts (but not allocation sites) that refer to type Custom $C$ are made to refer to AbstractCustomC instead.

- At this point, clones Custom $C_{1}, \cdots$, Custom $C_{n}$ of class CustomC can be introduced as a subclass of AbstractCustomC. Any allocation site of type Custom C may be updated to refer to any Custom $_{i}$.

The next section will discuss a number of optimizations that can be (independently) applied to each Custom $C_{i}$.

\section{Implementation}

We use the Gnosis framework for interprocedural context-sensitive analysis developed at IBM Research to compute all static analysis information that is needed for customization. Two nonstandard components of our analysis are:

- For customizable classes, each allocation site in user code is analyzed separately, but a single logical site represents all allocations in system code.

- Analysis is done in two passes: a conventional points-to analysis [20,13,21] is followed by a step in which additional data flow facts are introduced that model the type constraints due to method overriding, similar in spirit to [12].

Like many static whole-program analysis and transformation tools (e.g., [26]), Gnosis relies on the user to specify the behaviors of native library methods as well as any uses of reflection in order to compute a safe analysis result. (Note that specifying reflection entails specifying classes dynamically loaded via reflection.)

In order to gather profile information, the customization framework itself is used to replace the standard library classes created by an application with custom versions that gather profile information. This is subject to the usual limitations. In other words, we cannot gather profile information for container objects in cases where interface-compatibility constraints prevent us from applying customization. Usage statistics are gathered per allocation site and include:

1. A distribution of the construction-time sizes.

2. A distribution of the high-watermarks of the sizes of the container objects allocated at the site (i.e., the largest size of containers during their life-time).

3. Distributions of the container's size at method invocations (per method).

4. The hit-rates of search operations. 
5. The hit-rates of several caching schemes for optimizing search operations.

Distributions (1) and (2) are used as a basis for deciding on an initial allocation size and on lazy vs. eager allocation. Combined with (3), they are also used to determine whether providing special treatment for singleton containers is beneficial. Distribution (3) is also used to determine whether or not we want to optimize certain methods for specific sizes, such as empty or singleton containers. Distribution (4) is used to decide on whether or not search methods are to be optimized for succeeding or failing searches. Finally, (5) is used to decide on caching schemes.

Together with the static analysis results, the gathered statistics are used by an automated decision process to determine which optimizations, compared to the standard implementation, will be implemented in the custom versions. All decisions based on static analyses are binary decisions: either an optimization is safe or is not safe. Furthermore, all customization decisions that are based on profiles use thresholds, such as hit-rates for search operations, cache schemes, fractions of containers that remain empty or singletons, etc. As such, most of the profile-based decisions in our current implementation are simple binary decisions as well. The single exception is the decision on the initial size of underlying data structures of the containers. This takes the distribution of the high-watermarks of all allocated containers into consideration. We should note that all thresholds used are very high. For example, we use lazy allocation if $75 \%$ of the allocated containers remain empty or contain only one item. Similarly, caching schemes are used only if their hit-rates are $90 \%$ or higher.

The optimizations that are incorporated into custom classes include:

1. Caching the last retrieved items in a container using different caching schemes.

2. Lazy allocation of encapsulated data structures such as a Hashtable's array of hash-buckets,

3. Selecting a non-default initial size and growth-strategy for a container's underlying data structures, depending on the success-rate of retrieval operations, the distribution of the high-watermarks of the container sizes, etc.

4. Efficiently implementing frequently occurring corner cases such as container classes that often contain zero or one elements. For example, it is often possible to use a single, shared EmptyIterator whose hasNext() method always returns false.

5. Transforming instance fields into class fields if their values are identical for all objects allocated at some allocation site, or if the differences are non-critical.

6. Specialization of container classes for the type of objects stored in them, if static analysis can determine these types. Examples of such optimizations are Integer keys in Hashtables, for which we can store the int values instead, or Strings for which can exploit the fact that their hashcodes are cached.

7. Finalizing classes that have no subtypes in our program.

8. Removal of unnecessary synchronization. Currently, we only remove synchronization if an application is single-threaded. This is the case if the 
Thread.start() method is not reachable in the call-graph starting at the program entry point ${ }^{14}$.

These optimizations were chosen after carefully studying the existing implementations and conducting many experiments with special treatment for corner cases or access patterns. The inspiration for the optimizations originated from our own programming experience, from studying programs that heavily use container classes, and from searching the World Wide Web for manually crafted custom implementions.

In addition, static analysis information is used to detect situations where certain methods are never invoked on a container object that originates from a given allocation site $A$. This information is used to remove methods and fields from the custom class used at $A$. The bookkeeping fields used for implementing fail-fast iterators are an example of a situation where this is useful.

Java bytecode [16] is generated for each custom class by preprocessing a template implementation of a library class, and compiling the resulting source file to Java .class files. In the current implementation, this is done using the standard C-preprocessor. JikesBT ${ }^{15}$, a byte-code instrumentation tool developed at IBM Research, is used for the rewriting of the application's class files so that they refer to the generated custom classes.

\section{Experimental evaluation}

To evaluate our techniques, we measured the execution times and memory footprint of a number of Java applications on a workstation (hyperthreaded Pentium 4 at $2.8 \mathrm{GHz}, 1 \mathrm{~GB}$ RAM) running Linux 2.4.21 and two Java virtual machines: IBM's "j9" VM that is being distributed with IBM's WebSphere Studio Device Developer product and Sun's Hotspot Server 1.3.1 JVM. All measurements were performed using a maximum heap of $400 \mathrm{MB}$.

As our optimization technique aims at eliminating the overhead of using standard library implementations when such implementations are not optimal, we selected 7 benchmark programs that use the standard library classes in an atypical manner. Three programs are taken from the SPECjvm98 suite: _202_jess is an expert shell system,_209_db is a memory resident database, and _218_jack is a parser generator. The other benchmarks we include are HyperJ (an aspectoriented development tool), Jax [26], PmD (a open-source tool available from SourceForge for detecting programming errors) and a chess program developed at IBM. All execution times were measured using a harness: each program is executed 10 times within one invocation of the VM, and we report the fastest time of the 10 runs. With the exception of HyperJ, for which we had only one input data set available, all measurements were performed using larger data sets than the training sets used to collect profile information.

\footnotetext{
${ }^{14}$ Here, "Program entry point" refers to the entry point of the actual benchmark program, and not of the harness used for measuring execution time.

15 See www.alphaworks.ibm.com/tech/jikesbt
} 


\begin{tabular}{|c|c|c|c|c|c|c|c|c|}
\hline & \multicolumn{4}{|c|}{ Sun HotSpot(TM) Server 1.3.1 } & \multicolumn{4}{|c|}{ IBM J9 2.2} \\
\hline & orig & cust1 & cust2 & cust3 & orig & cust1 & cust2 & cust3 \\
\hline 202 jess & $1.92 \mathrm{~s}$ & $1.83 \mathrm{~s}(1.05)$ & $1.42 \mathrm{~s}(1.36)$ & $1.41 \mathrm{~s}(1.37)$ & $1.38 \mathrm{~s}$ & $1.45 \mathrm{~s}(0.95)$ & $1.21 \mathrm{~s}(1.14)$ & $1.21 \mathrm{~s}(1.15)$ \\
\hline _209_db & $15.04 \mathrm{~s}$ & $12.23 \mathrm{~s}(1.23)$ & $8.45 \mathrm{~s}(1.78)$ & $8.51 \mathrm{~s}(1.77)$ & $8.99 \mathrm{~s}$ & $8.63 \mathrm{~s}(1.04)$ & $6.48 \mathrm{~s}(1.39)$ & $6.48 \mathrm{~s}(1.39)$ \\
\hline 218 jack & $3.23 \mathrm{~s}$ & $3.18 \mathrm{~s}(1.02)$ & $2.28 \mathrm{~s}(1.42)$ & $2.24 \mathrm{~s}(1.44)$ & $1.88 \mathrm{~s}$ & $1.93 \mathrm{~s}(0.97)$ & $1.51 \mathrm{~s}(1.24)$ & $1.40 \mathrm{~s}(1.34)$ \\
\hline Jax & $21.46 \mathrm{~s}$ & $21.24 \mathrm{~s}(1.01)$ & $20.97 s$ (1.02) & $19.93 \mathrm{~s}(1.08)$ & $16.36 \mathrm{~s}$ & $15.04 \mathrm{~s}(1.09)$ & $15.00 \mathrm{~s}(1.09)$ & $14.90 \mathrm{~s}(1.10)$ \\
\hline HyperJ & $13.03 \mathrm{~s}$ & $12.77 \mathrm{~s}(1.02)$ & $11.04 \mathrm{~s}(1.18)$ & $10.83 \mathrm{~s}(1.20)$ & $10.04 \mathrm{~s}$ & $9.63 \mathrm{~s}(1.04)$ & $8.68 \mathrm{~s}(1.16)$ & $8.58 \mathrm{~s}(1.17)$ \\
\hline Chess & $18.07 \mathrm{~s}$ & $18.23 \mathrm{~s}(0.99)$ & $18.23 \mathrm{~s}(0.99)^{*}$ & $18.23 \mathrm{~s}(0.99)^{*}$ & $6.93 \mathrm{~s}$ & $6.59 \mathrm{~s}(1.05)$ & $6.59 \mathrm{~s}(1.05)^{*}$ & $6.59 \mathrm{~s}(1.05)^{*}$ \\
\hline Pmd & $5.43 \mathrm{~s}$ & $5.33 \mathrm{~s}(1.02)$ & $5.33 \mathrm{~s}(1.02)^{\star}$ & $5.33 \mathrm{~s}(1.02)^{\star}$ & $4.37 \mathrm{~s}$ & $3.76 \mathrm{~s}(1.16)$ & $3.76 \mathrm{~s}(1.16)^{\star}$ & $3.76 \mathrm{~s}(1.16)^{*}$ \\
\hline GEOMEAN & & $(1.05)$ & (1.23) & (1.24) & & (1.05) & (1.17) & (1.19) \\
\hline
\end{tabular}

Fig. 10. Execution times and speedups obtained through customization. The execution times presented are of the original programs (orig), the programs with customized container classes, but without synchronization elimination(cust1), the programs with customized containers classes of which we eliminated the synchronization where possible (cust2), and the programs with customized and desynchronized containers classes and desynchronized StringBuffers (cust3).

\begin{tabular}{|c|c|c|c|c|}
\hline & \multicolumn{2}{|c|}{ zipped archive } & \multicolumn{2}{|c|}{ heap size } \\
\hline & orig & cust3 & orig & cust 3 \\
\hline 202 jess & $173 \mathrm{~KB}$ & $175 \mathrm{~KB}(1.01)$ & $1.74 \mathrm{MB}$ & $1.77 \mathrm{MB}(0.99)$ \\
\hline $209 \_d b$ & $6 \mathrm{~KB}$ & $21 \mathrm{~KB}(3.68)$ & $9.57 \mathrm{MB}$ & $9.57 \mathrm{MB}(1.00)$ \\
\hline 218 jack & $70 \mathrm{~KB}$ & 95KB (1.35) & $15.78 \mathrm{MB}$ & $8.54 \mathrm{MB}(0.54)$ \\
\hline Jax & $582 \mathrm{~KB}$ & 615KB (1.06) & $44.23 \mathrm{MB}$ & 40.98MB (0.93) \\
\hline HyperJ & $1,767 \mathrm{~KB}$ & 1,821KB (1.03) & $44.42 \mathrm{MB}$ & $41.38 \mathrm{MB}(0.93)$ \\
\hline Chess & $135 \mathrm{~KB}$ & $151 \mathrm{~KB}(1.12)$ & $9.33 \mathrm{MB}$ & $9.29 \mathrm{MB}(1.00)$ \\
\hline Pmd & $498 \mathrm{~KB}$ & $525 \mathrm{~KB}(1.05)$ & $142.51 \mathrm{MB}$ & $123.72 \mathrm{MB}(0.87)$ \\
\hline GEOMEAN & & $(1.30)$ & & $(0.88)$ \\
\hline
\end{tabular}

Fig. 11. Archive size increase and memory footprint reduction resulting from customization.

In Figure 10 we report execution times of four versions of the benchmarks, that were customized in the exact same way for both VMs. ${ }^{16}$ The consequences of the customizations on memory consumption are depicted in Figure 11. An analysis of the obtained results and applied customizations reveals that:

- In _202_jess, the keys used in hashtables are either Strings or Integers, and on 2 of the hashtables all search operations fail. Depending on the VM used, customizing the Hashtable class for this usage pattern resulted in a $5 \%$ speedup or in a $5 \%$ slowdown. Eliminating synchronization, including the synchronization on very frequently used Vector objects, results in speedups between $15 \%$ and $37 \%$.

- In $\_209 \_d b, 99 \%$ of all consecutive retrieval operations on Vectors retrieve the same element (during what is essentially a column-major-order operation on

${ }^{16}$ In order to evaluate the customizations correctly, the original programs need to be executed with the original library classes before customization. Since all our customized classes are customized versions of IBM's jclMax implementation (which is distributed with IBM's WebSphere Studio Device Developer product), we enforced the use of the original jclMax classes on both VMs by prepending them to the boot classpath of the VMs. 
a row-major-order stored array of Vectors), and the application of a caching scheme results in a $23 \%$ speedup on the Sun VM. Additionally removing the synchronization on these Vectors results in a $77 \%$ speedup on the Sun VM, and a speedup of $39 \%$ on IBM's j9.

- In_228_jack, $99 \%$ of all search operations are on empty hashtables (of which a lot are allocated), or hashtables containing one element only. Using lazy allocation and eliminating the bookkeeping data for fail-safe iteration, we can reduce the heap memory consumption with $46 \%$. On Sun's VM, the resulting overhead in the retrieval operations can be compensated by optimizing the retrieval for the corner case of hashtables containing one element only. On IBM's j9, the overhead is almost compensated. Finally, removing synchronization results in speedups between 34 and $44 \%$.

- In HyperJ, the same situation occurs, and lazy allocation and the elimination of unnecessary bookkeeping data for the hot allocation sites results in speedups around $2-4 \%$ if no synchronization is eliminated, and $17-20 \%$ if synchronization is eliminated. Memory consumption drops with $7 \%$. Especially for this benchmark, we should note that our customization results are obtained on top of the already applied manual fine-tuning by the programmers via construction time parameterization.

- In Jax, most containers remain very small, and adapting the initial container size to reflect that results in speedups ranging between 1 and $3 \%$. Memory consumption as a result drops with $7 \%$.

- In PmD, the vast majority of a huge number of allocated HashMaps remains empty or contains only one element. Lazy allocation, the elimination of bookkeeping data for fail-safe iteration and the optimization of access methods result in speedups of 2 to $16 \%$, and a reduction of the allocated memory with $13 \%$. Since PmD contains a multi-threaded GUI front-end, no synchronization was removed.

- In the chess program, enumerations (over piece positions) stored in hashtables occur very frequently. Optimizing the number of hash-buckets for the number of positions (which seemed limited to the number of pieces on a board) resulted in speedups between 2 and 16\%, depending on the VM. Like Pmd, the chess program contains a multi-threaded front-end. As a result, we did not yet try to eliminate any synchronization.

On average, the customizations excluding the elimination of synchronization result in speedups of $5 \%$ on both VMs. By additionally eliminating synchronization on container classes and StringBuffers in the single-threaded programs, an average speedup of $19-24 \%$ can be obtained. The elimination of synchronization is therefore clearly the dominant optimization, in particular the elimination of synchronization on container classes. There are exceptions to this trend however: for Jax the speedup following synchronization removal is insignificant on j9, and for 218_jack the removal of synchronization on StringBuffers is much more significant (10\% additional speedup) than for the other programs.

The raw execution times shown in Figure 10 indicate that the two VMs have somewhat different performance characteristics. It is therefore no surprise that 
the obtained speedups are different for each VM as well. This indicates that the decision logic used for the customization should be made parameterizable for specific VMs.

Finally, we should note that program archive size increases only by a small amount because of the customization: as indicated in Figure 11 at most 54KB is added to the archives, for HyperJ. For all but the smallest programs the zipped archives grow by $12 \%$ or less.

\section{Related Work}

Yellin [28] and Högstedt et al. [14] discuss techniques for automatically selecting optimal component implementations. In [28], selection takes place at run-time, and based on on-line profiling only, while in [14] off-line profiling is used as well. In both cases, the component developer is required to provide all component versions up front, making them less viable when many orthogonal implementation decisions exist, as is the case in the present paper. Unlike our work, the approaches of $[28,14]$ do not require static analysis because programs are correct by construction. In our setting, static analysis is needed to guarantee typecorrectness in cases where objects are exchanged with the standard libraries (or other components). However, as the approaches of $[28,14]$ do not rely on static analysis, they are incapable of eliminating functionality from classes.

Schonberg et al. [22] discuss techniques for automatically selecting concrete data structures to implement the abstract data types set and map in SETL programs. Depending on whether or not iterators and set-theoretic operations such as union and intersection are applied to abstract data types, their optimizing compiler selects an implementation from a predetermined collection of implementations in which sets are represented as linked lists, hashtables, or bitvectors.

Instead of composing complex data structures from simpler ones, as is done with $\mathrm{C}++$ templates or Ada generics, Sirkin et al. [24] describe how custom, optimized data structures can be generated automatically from a relatively simple specification that consists of a composition of iterators and access methods defined in a very generic container interface. To some extent this data structure compilation resembles our approach to eliminate unnecessary fields. But whereas we remove unnecessary fields when static analysis detects that access methods in the standard interfaces are not used in a program, they add fields to a data structure to allow an efficient implementation of those access methods occurring in the specification provided by the programmer.

Transformational programming [2] is a programming methodology based on top-down stepwise program refinement. Here, the programmer specifies a program in terms of very high-level constructs without worrying about efficiency. This program is made more efficient by automatically applying a sequence of finite difference transformations or by applying incremental refinement $[9,19]$.

There is a large body of work on automatic optimization of data structures in specific domains (e.g., linear algebra kernels). For example, the Berkeley Bench- 
marking and Optimization Group (see http://bebop.cs.berkeley .edu) studies issues related to optimization and data structure selection for sparse matrix multiplication problems. In the same domain, Yotov et al. [29] compare empirical and model-driven approaches for selecting customized data structures.

Our customization approach may be seen as a type of generative programming [8], in which the program analysis and rewriting tools needed for our approach, the instrumented classes to collect profiling information, the templates to generate custom classes and the decision logic can be seen as an active library [27]. Active libraries are highly parameterizable libraries that require implementation techniques beyond what is provided by traditional compilers.

There is also work on optimizations that can be applied to specific containers such as hashtables. Beckmann and Wang [1] discuss the optimization of hashtables by prefetching the objects that are most likely to be searched for, and Friedman et al. [11] discuss the optimization of the maximal access time of hashtables to improve real-time behavior by, e.g., incrementalizing rehash operations.

The elimination of synchronization has been a very popular research subject (see for example [5]). To the best of our knowledge, all currently known solutions are either based on manual rewriting of a program to remove the synchronization or on bytecode annotations that can only be read by adapted VMs. Our approach of automatically replacing the allocation of standard types with the allocation of customized types allows to automate the existing techniques for synchronization elimination without requiring special VM support.

Based on partial evaluation and the use of aspects [15], Schultz et al. [23] specialize methods for (partially known) input data, resulting in average speed-ups by a factor of 3 . While such specialization can only exploit knowledge of (properties of) parts of the input data, our customization approach is able to exploit known access patterns of otherwise unknown input data as well. Furthermore, whereas we were able to apply our approach in large real-life applications, we have not seen any indication of the scalability of program specialization through partial evaluation. The bytecode size of the largest benchmark program used by Schultz et al. [23], e.g., is only 4914 bytes. With respect to the use of aspects, we should note that some of our customizations, such as adding a cache to speed-up hashtable accesses, could be implemented with aspects as well. But other customizations, such as the removal of unused bookkeeping fields from container types, can obviously not.

A limited form of program specialization is customization, as introduced by Chambers and Ungar [4]. They used the term customization to denote dynamic method cloning in SELF compilers, in which a separate clone is generated and optimized for each of the receiver types of a method. Other cloning techniques have been applied on more traditional programming languages such as Fortran as well [7], albeit not to optimize procedures for receiver types but rather to allow other optimizations such as loop unrolling.

Besides specializing methods or procedures for statically known (properties of) input data, value profiling $[3,6]$ has been used to produce code that is optimized for likely occurring situations. This is somewhat comparable to our profil- 
ing based optimizations. But whereas value profiling aims at lower level compiler optimization, e.g., by propagating constant values, our approach aims for algorithmic optimizations (such as caching).

During Java's short lifetime, the standard Java Library classes have been extended on several occasions. For example, the original synchronized Hashtable type present in JDK1.0 was complemented with the unsynchronized type HashMap in JDK1.2 Similarly, the type StringBuilder is added in JDK1.5 as an unsynchronized sibling of the synchronized type StringBuffer. Unlike the approach we presented in this paper, such extensions of the standard library classes can only accommodate a limited number of different implementations for each abstract data type. Moreover, such extensions do not remove the burden from the programmer to choose an actual implementation for his program. One of the main benefits of our approach is its transparency for the programmer.

Type constraints were introduced [18] as a means to check whether a program conforms to a language's type system. If a program satisfies all type constraints, no type violations will occur at run-time (e.g., no method $m(\cdots)$ is invoked on an object whose class does not define or inherit $m(\cdots))$. Type constraints were recently used to check the preconditions and determine the allowable source-code modifications associated with generalization-related refactorings [25]. The problem of determining where custom versions of library classes may be introduced is very similar to the problem of determining declarations that can be updated by the Extract InTERFACE refactoring. However, in [25] the type of a declaration is only replaced with one of its supertypes, whereas our work is unique in the sense that custom classes appear in a different branch of the class hierarchy.

\section{Conclusions and Future Work}

We have presented an automated approach for customizing Java container classes that relies on static analysis for determining where custom container classes may be introduced in an application, and on profile information for determining what optimizations are likely to be profitable. The approach was evaluated on a set of benchmark applications, and we measured speedups of up to $78 \%$, averaging at 19-24\%. The memory footprint reductions we measured range from -1 to $46 \%$, averaging at $12 \%$. The cost of the applied customizations in terms of code size is limited to $12 \%$ for all of the smallest programs we evaluated.

We plan to develop a more precise formal treatment of the properties of our algorithm for determining where custom allocation sites may be introduced. Other topics for future work include more advanced program transformations (e.g, replacing a Hashtable with an extra field in each key object that stores a reference to its corresponding value), applications to other library classes and the use of escape analysis to determine where unnecessary synchronizations can be removed from multi-threaded programs. 


\section{Acknowledgement}

Bjorn De Sutter is supported by the Fund for Scientific Research - Flanders (FWO) as a postdoctoral researcher. The research reported in this paper took place during his stay at IBM T.J. Watson Research Center in Hawthorne, N.Y. This stay was also supported by the FWO.

\section{References}

1. Beckmann, B., And WAng, X. Adaptive prefetching Java objects. manuscript.

2. CAI, J., AND PAige, R. Towards increased productivity of algorithm implementation. In Proc. 1st ACM SIGSOFT symposium on Foundations of software engineering (1993), pp. 71-78.

3. Calder, B., Feller, P., And Eustace, A. Value profiling. In Proceedings of the 30th annual ACM/IEEE International Symposium on Microarchitecture (ISCA97) (1997), pp. 259-269.

4. Chambers, C., And Ungar, D. Customization: optimizing compiler technology for SELF, a dynamically-typed object-oriented programming language. ACM SIGPLAN Notices 24, 7 (july 1989), 146-160.

5. Choi, J.-D., Gupta, M., Serrano, M. J., Sreedhar, V. C., and Midkiff, S. P. Stack allocation and synchronization optimizations for java using escape analysis. ACM Transactions on Programming Languages and Systems 25, 6 (2003), 876-910.

6. Chung, E.-Y., Benini, L., And De Micheli, G. Automatic source code specialization for energy reduction. In Proceedings of the 2001 International Symposium on Low Power Electronics and Design (ISPLED01) (2001), pp. 80-83.

7. Cooper, K., Hall, M. W., And Kennedy, K. A methodology for procedure cloning. Computer Languages (may 1995).

8. Czarnecki, K., And Eisenecker, U. Generative Programming: Methods, Techniques, and Applications. Addison-Wesley, 1999.

9. Dewar, R. K., Arthur, Liu, S.-C., Schwartz, J. T., and Schonberg, E. Programming by refinement, as exemplified by the SETL representation sublanguage. ACM Transactions on Programming Languages and Systems (TOPLAS) 1, 1 (1979), 27-49.

10. Fowler, M. Refactoring. Improving the Design of Existing Code. Addison-Wesley, 1999.

11. Friedman, S., Leidenfrost, N., Brodie, B., And Cytron, R. Hashtables for embedded and real-time systems. In IEEE Real-Time Embedded System Workshop (2001)

12. Glew, N., And Palsberg, J. Type-safe method inlining. In Proc. 16th European Conference on Object-Oriented Programming (2002), pp. 525-544.

13. Hind, M., AND Pioli, A. Evaluating the effectiveness of pointer alias analyses. Science of Computer Programming 39, 1 (2001), 31-55.

14. Högstedt, K., D., K., Rajan, V., Roth, T., Sreedhar, V., Wegman, M., AND WAng, N. The autonomic performance prescription. Available from the author at wegman@watson.ibm.com.

15. Kiczales, G., Lamping, J., Menhdhekar, A., Maeda, C., Lopes, C., LoINGTIER, J.-M., AND IRWIn, J. Aspect-oriented programming. In Proceedings of the European Conference on Object-Oriented Programming (ECOOP97) (1997), vol. 1241, Springer-Verlag, pp. 220-242. 
16. Lindholm, T., And Yellin, F. The Java Virtual Machine Specification. AddisonWesley, 1997.

17. PAlsberg, J. Type-based analysis and applications. In Proc. ACM Workshop on Program Analysis for Software Tools and Engineering (PASTE'01) (Snowbird, UT, 2001), pp. 20-27.

18. Palsberg, J., And Schwartzbach, M. Object-Oriented Type Systems. John Wiley \& Sons, 1993.

19. PAvlOvic, D., AND Smith, D. Software development by refinement. In UNU/IIST 10th Anniversary Colloqium, Formal Methods at the Crossroads: From Panaea to Foundational Support. Springer-Verlag, 2003.

20. Rountev, A., Milanova, A., And Ryder, B. Points-to analysis for Java using annotated constraints. In Proc. 16th Annual Conf. on Object-Oriented Programming Systems, Languages, and Applications (OOPSLA'01) (Tampa Bay, FL, 2001), pp. $43-55$.

21. RYDER, B. G. Dimensions of precision in reference analysis of object-oriented programming languages. In Proc. 12th International Conference on Compiler Construction (CC 2003) (Warsaw, Poland, April 2003), pp. 126-137.

22. Schonberg, E., Schwartz, J., And Sharir, M. An automatic technique for selection of data representations in SETL programs. ACM Transactions on Programming Languages and Systems 3, 2 (April 1981), 126-143.

23. Schultz, U. P., Lawall, J. L., And Consel, C. Automatic program specialization for java. ACM Transactions on Programming Languages and Systems 25, 4 (2003), 452-499.

24. Sirkin, M., Batory, D., And Singhal, V. Software components in a data structure precompiler. In Proceedings of the 15th International Conference on Software Engineering (ICSE97) (1997), pp. 437-446.

25. TiP, F., Kieżun, A., AND BÄUmer, D. Refactoring for generalizations using type constraints. In Proc. 18th Annual Conf. on Object-Oriented Programming Systems, Languages, and Applications (OOPSLA'03) (2003).

26. Tip, F., Sweeney, P. F., Laffra, C., Eisma, A., and Streeter, D. Practical extraction techniques for Java. ACM Transactions on Programming Languages and Systems 24, 6 (2002), 625-666.

27. Veldhuizen, T. L., And Gannon, D. Active libraries: Rethinking the roles of compilers and libraries. In Proceedings of the SIAM Workshop on Object Oriented Methods for Inter-operable Scientific and Engineering Computing (OO'98) (1998).

28. Yellin, D. Competitive algorithms for the dynamic selection of component implementations. IBM Systems Journal 42, 1 (January 2003), 85-97.

29. Yotov, K., Li, X., Ren, G., Cibulskis, M., DeJong, G., Garzaran, M., Padua, D., Pingali, K., Stodghill, P., and Wu, P. A comparison of empirical and model-driven optimization. In Proc. ACM SIGPLAN 2003 conference on Programming language design and implementation (2003), pp. 63-76. 